TRANSACTIONS OF THE

AMERICAN MATHEMATICAL SOCIETY

Volume 362, Number 8, August 2010, Pages 4063-4076

S 0002-9947(10)04741-0

Article electronically published on March 24, 2010

\title{
COMPACT COMPLETE MINIMAL IMMERSIONS IN $\mathbb{R}^{3}$
}

\author{
ANTONIO ALARCÓN
}

\begin{abstract}
In this paper we find, for any arbitrary finite topological type, a compact Riemann surface $\mathcal{M}$, an open domain $M \subset \mathcal{M}$ with the fixed topological type, and a conformal complete minimal immersion $X: M \rightarrow \mathbb{R}^{3}$ which can be extended to a continuous map $X: \bar{M} \rightarrow \mathbb{R}^{3}$, such that $\left.X\right|_{\mid \partial M}$ is an embedding and the Hausdorff dimension of $X(\partial M)$ is 1 .

We also prove that complete minimal surfaces are dense in the space of minimal surfaces spanning a finite set of closed curves in $\mathbb{R}^{3}$, endowed with the topology of the Hausdorff distance.
\end{abstract}

\section{INTRODUCTION}

In 1996, Nadirashvili [14] constructed the first example of a complete bounded minimally immersed disk in $\mathbb{R}^{3}$, giving a counterexample to the Calabi-Yau conjecture [4]. Calabi conjectured that a complete minimal surface in $\mathbb{R}^{3}$ must be unbounded. Nadirashvili's work seemed to be the end point of a classical problem. However, his methods and ideas were the beginning of a significant development in the construction of complete bounded minimal surfaces. Much work has been done to study the topology and limit sets of complete bounded minimal immersions (see for instance [8, 10, 11]).

Assume $M$ is an open proper subdomain of a compact Riemann surface and let $X: M \rightarrow \mathbb{R}^{3}$ be a conformal complete minimal immersion. Denote by $\Gamma$ the limit set of the surface $X(M)$; i.e., $\Gamma \subset \mathbb{R}^{3}$ is the limit set of $X(p)$ for $p \rightarrow \partial M$. It was proven by Ferrer, Martín and the author 2 that any open surface of finite topology can be properly and minimally immersed in any convex domain of $\mathbb{R}^{3}$ or any bounded and smooth domain of $\mathbb{R}^{3}$. In particular, its limit set is contained in the boundary of that domain. Moreover, the following deep result was recently proved by Ferrer, Martín and Meeks [7]: Let $D$ be a domain which is convex (possibly $D=\mathbb{R}^{3}$ ) or smooth and bounded; given any open surface $M$, there exists a complete proper minimal immersion $X: M \rightarrow D$.

On the other hand, some nonexistence theorems imposed some limits to the theory. Martín, Meeks and Nadirashvili 9 showed the existence of a domain $D \subset$ $\mathbb{R}^{3}$ for which there are no complete properly immersed in $D$ minimal surfaces. Furthermore, there exist requirements for the limit set of compact complete minimal immersions, according to the following definition.

Received by the editors November 16, 2007.

2010 Mathematics Subject Classification. Primary 53A10; Secondary 53C42, 49Q05.

Key words and phrases. Complete minimal surfaces, Plateau problem.

The author was partially supported by Spanish MEC-FEDER Grant MTM2007-61775 and Regional J. Andalucía Grant P09-FQM-5088.

(C)2010 American Mathematical Society Reverts to public domain 28 years from publication 
Definition 1. By a compact minimal immersion we mean a minimal immersion $X: M \rightarrow \mathbb{R}^{3}$, where $M$ is an open region of a compact Riemann surface $\mathcal{M}$, and such that $X$ can be extended to a continuous map $X: \bar{M} \rightarrow \mathbb{R}^{3}$.

If we take $M=\mathbb{D}$, the unit disk of $\mathbb{C}$, and if we assume that an immersion $X: M \rightarrow \mathbb{R}^{3}$ is complete and compact, then its limit set $\Gamma$ cannot be a subset of the boundary of a cube in $\mathbb{R}^{3}[15$, i.e., $X$ cannot be proper in a cube. In fact, if such an immersion is proper in a regular domain $C \subset \mathbb{R}^{3}$, then the second fundamental form of the surface $\partial C$ at any point of the limit set must be nonnegatively definite 3. Moreover, $C$ cannot be a polyhedron 3 .

The study of the Calabi-Yau problem gave rise to new lines of work and techniques. Among other things, these new ideas established a surprising relationship between the theory of complete minimal surfaces in $\mathbb{R}^{3}$ and the Plateau problem. This problem consists of finding a minimal surface spanning a given closed curve in $\mathbb{R}^{3}$, and it was solved independently by Douglas [6] and Radó [16, for any Jordan curve. The link between complete minimal surfaces and the Plateau problem is the existence of compact complete minimal immersions in $\mathbb{R}^{3}$. Martín and Nadirashvili [12] constructed compact complete conformal minimal immersions $X: \mathbb{D} \rightarrow \mathbb{R}^{3}$ such that $X_{\mid \partial \mathbb{D}}$ is an embedding and $X\left(\mathbb{S}^{1}\right)$ is a Jordan curve with Hausdorff dimension 1. Furthermore, they showed that the set of Jordan curves $X\left(\mathbb{S}^{1}\right)$ constructed by the above procedure is dense in the space of Jordan curves of $\mathbb{R}^{3}$ with the Hausdorff distance.

The aim of this paper is to join the techniques used in the construction of complete bounded minimal surfaces with arbitrary finite topology, and those used to construct complete minimal disks spanning Jordan curves, in order to prove the following result.

Theorem. There exist compact complete minimal immersions $X: M \rightarrow \mathbb{R}^{3}$ of arbitrary finite topological type and such that the extended map $X: \bar{M} \rightarrow \mathbb{R}^{3}$ satisfies that $X_{\mid \partial M}$ is an embedding and the Hausdorff dimension of $X(\partial M)$ is 1 .

Moreover, for any finite family $\Sigma$ of closed curves in $\mathbb{R}^{3}$ which admits a solution to the Plateau problem, and for any $\xi>0$, there exists a minimal immersion $X: M \rightarrow \mathbb{R}^{3}$ with the above conditions and such that $\delta^{H}(\Sigma, X(\partial M))<\xi$, where $\delta^{H}$ means the Hausdorff distance.

Unfortunately, our arguments do not guarantee that the boundary curves of $M$ are Jordan curves in $\mathcal{M}$. Therefore, although $X_{\mid \partial M}$ is an embedding, we cannot assert that $X(\partial M)$ consists of Jordan curves. It is also important to notice that $X$ cannot be an embedding following the recent result by Colding and Minicozzi [5] which asserts that any complete embedded minimal surface in $\mathbb{R}^{3}$ with finite topology is proper in $\mathbb{R}^{3}$. In particular, such a surface must be unbounded, and so it cannot be contained in a compact set.

In this paper we also prove the following density result for compact complete minimal immersions in $\mathbb{R}^{3}$.

Theorem. Complete minimal surfaces are dense in the space of minimal surfaces spanning a finite set of closed curves in $\mathbb{R}^{3}$, endowed with the topology of the Hausdorff distance. 


\section{Preliminaries}

This section is devoted to briefly summarizing the notation and results that we use in the paper.

2.1. Riemann surfaces background. Throughout the paper we work on a compact Riemann surface endowed with a Riemannian metric. We consider that the following data are fixed.

Definition 2. Let $M^{\prime}$ be a compact Riemann surface of genus $\sigma \in \mathbb{N} \cup\{0\}$, and let $d s^{2}$ be a Riemannian metric in $M^{\prime}$.

Consider a subset $W \subset M^{\prime}$ and a Riemannian metric $d \tau^{2}$ in $W$. Given a curve $\alpha$ in $W$, by length $d \tau(\alpha)$ we mean the length of $\alpha$ with respect to the metric $d \tau^{2}$. Moreover, we define:

- $\operatorname{dist}_{(W, d \tau)}(p, q)=\inf \left\{\operatorname{length}_{d \tau}(\alpha) \mid \alpha:[0,1] \rightarrow W, \alpha(0)=p, \alpha(1)=q\right\}$, for any $p, q \in W$

- $\operatorname{dist}_{(W, d \tau)}\left(T_{1}, T_{2}\right)=\inf \left\{\operatorname{dist}_{(W, d \tau)}(p, q) \mid p \in T_{1}, q \in T_{2}\right\}$, for any $T_{1}, T_{2} \subset W$;

- $\operatorname{diam}_{d \tau}(W)=\sup \left\{\operatorname{dist}_{(W, d \tau)}(p, q) \mid p, q \in W\right\}$.

Throughout the paper, we work with a domain $W$ in $M^{\prime}$ and a conformal minimal immersion $Y: \bar{W} \rightarrow \mathbb{R}^{3}$. Then, by $d s_{Y}^{2}$ we mean the Riemannian metric induced by $Y$ in $\bar{W}$. We also write $\operatorname{dist}_{(\bar{W}, Y)}\left(T_{1}, T_{2}\right)$ instead of $\operatorname{dist}_{\left(\bar{W}, d s_{Y}\right)}\left(T_{1}, T_{2}\right)$, for any sets $T_{1}$ and $T_{2}$ in $\bar{W}$.

For $\mathrm{E} \in \mathbb{N}$, consider $\mathbb{D}_{1}, \ldots, \mathbb{D}_{\mathrm{E}} \subset M^{\prime}$ open disks so that $\left\{\gamma_{i}:=\partial \mathbb{D}_{i}\right\}_{i=1}^{\mathrm{E}}$ are analytic Jordan curves and $\overline{\mathbb{D}}_{i} \cap \overline{\mathbb{D}}_{j}=\emptyset$ for all $i \neq j$.

Definition 3. Each curve $\gamma_{i}$ will be called a cycle on $M^{\prime}$ and the family $\mathcal{J}=$ $\left\{\gamma_{1}, \ldots, \gamma_{\mathrm{E}}\right\}$ will be called a multicycle on $M^{\prime}$. We denote by $\operatorname{Int}\left(\gamma_{i}\right)$ the disk $\mathbb{D}_{i}$, for $i=1, \ldots$, E. We also define $M(\mathcal{J})=M^{\prime} \backslash\left(\bigcup_{i=1}^{\mathrm{E}} \overline{\operatorname{Int}\left(\gamma_{i}\right)}\right)$.

Given $\mathcal{J}=\left\{\gamma_{1}, \ldots, \gamma_{\mathrm{E}}\right\}$ and $\mathcal{J}^{\prime}=\left\{\gamma_{1}^{\prime}, \ldots, \gamma_{\mathrm{E}}^{\prime}\right\}$ two multicycles on $M^{\prime}$ we write $\mathcal{J}^{\prime}<\mathcal{J}$ if $\overline{\operatorname{Int}\left(\gamma_{i}\right)} \subset \operatorname{Int}\left(\gamma_{i}^{\prime}\right)$ for $i=1, \ldots$, E. Notice that $\mathcal{J}^{\prime}<\mathcal{J}$ implies $\overline{M\left(\mathcal{J}^{\prime}\right)} \subset$ $M(\mathcal{J})$.

Let $\mathcal{J}=\left\{\gamma_{1}, \ldots, \gamma_{\mathrm{E}}\right\}$ be a multicycle on $M^{\prime}$. For $\epsilon>0$ small enough, we can consider the multicycle $\mathcal{J}^{\epsilon}=\left\{\gamma_{1}^{\epsilon}, \ldots, \gamma_{\mathrm{E}}^{\epsilon}\right\}$, where by $\gamma_{i}^{\epsilon}$ we mean the cycle satisfying $\overline{\operatorname{Int}\left(\gamma_{i}\right)} \subset \operatorname{Int}\left(\gamma_{i}^{\epsilon}\right)$ and $\operatorname{dist}_{\left(M^{\prime}, d s\right)}\left(q, \gamma_{i}\right)=\epsilon$ for all $q \in \gamma_{i}^{\epsilon}$ and $i=1, \ldots$, E. Notice that $\mathcal{J}^{\epsilon}<\mathcal{J}$.

Definition 4. Let $K$ be a compact subset in the Riemann surface $M^{\prime}, n \in \mathbb{N}$, and let $f: K \rightarrow \mathbb{R}^{3}$ be a continuous embedding. We define

$$
\Psi(K, f, n):=\frac{1}{2 n^{2}} \inf \left\{\|f(p)-f(q)\| \mid p \neq q \in K, \operatorname{dist}_{\left(M^{\prime}, d s\right)}(p, q)>\frac{1}{n}\right\} .
$$

Notice that $\Psi(K, f, n)>0$.

2.2. Minimal surfaces background. The theory of complete minimal surfaces is closely related to the theory of Riemann surfaces. This is due to the fact that any such surface is given by a triple $\Phi=\left(\Phi_{1}, \Phi_{2}, \Phi_{3}\right)$ of holomorphic 1-forms defined on some Riemann surface such that

$$
\begin{gathered}
\Phi_{1}^{2}+\Phi_{2}^{2}+\Phi_{3}^{2}=0, \\
\left\|\Phi_{1}\right\|^{2}+\left\|\Phi_{2}\right\|^{2}+\left\|\Phi_{3}\right\|^{2} \neq 0,
\end{gathered}
$$


and all periods of the $\Phi_{j}$ are purely imaginary. Here we consider $\Phi_{i}$ to be a holomorphic function times $d z$ in a local parameter $z$. Then the minimal immersion $X: M \rightarrow \mathbb{R}^{3}$ can be parameterized by $z \mapsto \operatorname{Re} \int^{z} \Phi$. The above triple is called the Weierstrass representation of the immersion $X$. Usually, the first requirement (2.1) (which ensures the conformality of $X$ ) is guaranteed by introducing the formulas

$$
\Phi_{1}=\frac{1}{2}\left(1-g^{2}\right) \eta, \quad \Phi_{2}=\frac{\mathrm{i}}{2}\left(1+g^{2}\right) \eta, \quad \Phi_{3}=g \eta,
$$

with a meromorphic function $g$ (the stereographic projection of the Gauss map) and a holomorphic 1-form $\eta$. The metric of $X$ can be expressed as

$$
d s_{X}^{2}=\frac{1}{2}\|\Phi\|^{2}=\left(\frac{1}{2}\left(1+|g|^{2}\right)\|\eta\|\right)^{2} .
$$

2.2.1. The López-Ros transformation. The proof of Lemma 1 exploits what has come to be called the López-Ros transformation. If $M$ is a Riemann surface and $(g, \eta)$ are the Weierstrass data of a minimal immersion $X: M \rightarrow \mathbb{R}^{3}$, we define on $M$ the data

$$
\widetilde{g}=\frac{g}{h}, \quad \widetilde{\eta}=\eta \cdot h,
$$

where $h: M \rightarrow \mathbb{C}$ is a holomorphic function without zeros. If the periods of this new Weierstrass representation are purely imaginary, then it defines a minimal immersion $\tilde{X}: M \rightarrow \mathbb{R}^{3}$. This method provides us with a powerful and natural tool for deforming minimal surfaces. From our point of view, the most important property of the resulting surface is that the third coordinate function is preserved. Note that the intrinsic metric is given by (2.2) as

$$
d s_{\widetilde{X}}^{2}=\left(\frac{1}{2}\left(|h|+\frac{|g|^{2}}{|h|}\right)\|\eta\|\right)^{2} .
$$

This means that we can increase the intrinsic distance in a prescribed compact of $M$, by using suitable functions $h$.

2.3. Hausdorff distance. Throughout the paper we denote by $\delta^{H}(C, D)$ the Hausdorff distance between two nonempty compact subsets of $\mathbb{R}^{n}, C$ and $D$, i.e.,

$$
\delta^{H}(C, D)=\max \left\{\sup _{x \in C} \inf _{y \in D}\|x-y\|, \sup _{y \in D} \inf _{x \in C}\|x-y\|\right\} .
$$

2.4. Preliminary lemma. Consider a multicycle $\mathcal{I}=\left\{\Sigma_{1}, \ldots, \Sigma_{\mathrm{E}}\right\}$ on the compact surface $M^{\prime}$. We denote $M=\overline{M(\mathcal{I})}$. For any $i \in\{1, \ldots, \mathrm{E}\}$, let $\beta_{i}: \Sigma_{i} \rightarrow \mathbb{R}^{3}$ be an analytic curve. Finally, given $\mathcal{T}\left(\Sigma_{i}\right)$ a tubular neighborhood of $\Sigma_{i}$ in $M$, we denote by $\mathrm{P}_{i}: \mathcal{T}\left(\Sigma_{i}\right) \rightarrow \Sigma_{i}$ the natural projection.

Taking this setup into account, the following result was essentially proved by Ferrer, Martín and the author [2, Lemma 3]. It concentrates on most of the technical computations required in the main lemma of this paper (Lemma 2).

Lemma 1. Consider $\mathcal{J}=\left\{\gamma_{1}, \ldots, \gamma_{\mathrm{E}}\right\}$ a multicycle on $M, X: \overline{M(\mathcal{J})} \rightarrow \mathbb{R}^{3}$ a conformal minimal immersion, $p_{0}$ a point in $M(\mathcal{J})$, and $r>0$, such that

- $X\left(p_{0}\right)=0$

- $\gamma_{i} \subset \mathcal{T}\left(\Sigma_{i}\right)$, for any $i \in\{1, \ldots, \mathrm{E}\}$;

- $\left\|X(p)-\beta_{i}\left(\mathrm{P}_{i}(p)\right)\right\|<r$, for all $p \in \gamma_{i}$ and for all $i=1, \ldots, \mathrm{E}$. 
Then, for any $s>0$, and any $\epsilon>0$ so that $p_{0} \in M\left(\mathcal{J}^{\epsilon}\right)$, there exist a multicycle $\widetilde{\mathcal{J}}=\left\{\widetilde{\gamma}_{1}, \ldots, \widetilde{\gamma}_{\mathrm{E}}\right\}$ and a conformal minimal immersion $\widetilde{X}: \overline{M(\widetilde{\mathcal{J}})} \rightarrow \mathbb{R}^{3}$ satisfying:

(L1,1) $\tilde{X}\left(p_{0}\right)=0$.

(L1,2) $\widetilde{X}\left(\widetilde{\gamma}_{i}\right)$ is a Jordan curve, $\forall i=1, \ldots, \mathrm{E}$.

(L1,3) $\widetilde{\gamma}_{i} \subset \mathcal{T}\left(\Sigma_{i}\right)$, for all $i=1, \ldots, \mathrm{E}$.

(L1,4) $\mathcal{J}^{\epsilon}<\widetilde{\mathcal{J}}<\mathcal{J}$.

(L1,5) $s<\operatorname{dist} \frac{}{(M(\widetilde{\mathcal{J}}), \widetilde{X})}(p, \widetilde{\mathcal{J}}), \forall p \in \mathcal{J}^{\epsilon}$.

(L1,6) $\|\tilde{X}-X\|<\epsilon$ in $\overline{M\left(\mathcal{J}^{\epsilon}\right)}$.

(L1,7) $\left\|\widetilde{X}(p)-\beta_{i}\left(\mathrm{P}_{i}(p)\right)\right\|<R=\sqrt{4 s^{2}+r^{2}}+\epsilon, \forall p \in \widetilde{\gamma}_{i}, \forall i=1, \ldots$, E.

Actually, the above lemma improves Lemma 3 in 22. The improvement consists of the addition of Statement (L1,2). Checking the proof given in 2, we notice that this assertion can be trivially guaranteed. Item (L1,2) holds up to infinitesimal variations of $\widetilde{\mathcal{J}}$. These variations can be done without losing the remaining properties.

2.4.1. Sketch of the proof of Lemma 1, The proof is inspired by a technique introduced by Martín and Nadirashvili in [12. See [2, §5.1] for further details.

First of all, fix $\omega$ a meromorphic differential on $M^{\prime}$ so that $\omega$ has neither zeroes nor poles on $\overline{M(\mathcal{J})}$. Then it is well known that $\|\omega\|^{2}$ is a flat Riemannian metric on $\overline{M(\mathcal{J})}$. Assume $d s^{2}=\|\omega\|^{2}$.

Let $\zeta \in] 0, \epsilon\left[\right.$ be small enough so that $\gamma_{i}^{\zeta} \subset \mathcal{T}\left(\Sigma_{i}\right)$, for $i=1, \ldots$, E. Consider $N \in \mathbb{N}$ such that $2 / N<\zeta$.

Throughout the sketch of the proof, a set of real positive constants not depending on $N$ will appear. The symbol 'const' will denote these different constants.

We consider an order relation in the set $I \equiv\{1, \ldots, 2 N\} \times\{1, \ldots, \mathrm{E}\}$. We say that $(j, l)>(i, k)$ if one of the following situations holds: $l=k$ and $j>i$, or $l>k$.

The next step consists of the construction of a Nadirashvili's type labyrinth along the cycle $\gamma_{k}, k=1, \ldots$, E. Let $\left\{v_{1, k}, \ldots, v_{2 N, k}\right\}$ be a set of points in the curve $\gamma_{k}$ that divide $\gamma_{k}$ into $2 N$ equal parts (i.e., curves with the same length). Following the normal projection, we can transfer the above partition to the curve $\gamma_{k}^{2 / N}:\left\{v_{1, k}^{\prime}, \ldots, v_{2 N, k}^{\prime}\right\}$. We define the following sets:

- $L_{i, k}$ is the minimizing geodesic in $\left(\overline{M(\mathcal{J})}, d s^{2}\right)$ joining $v_{i, k}$ and $v_{i, k}^{\prime},(i, k) \in I$.

- $\mathcal{G}_{j, k}=\gamma_{k}^{j / N^{3}}, \forall j=0, \ldots, 2 N^{2}$ (recall that $\gamma_{k}^{j / N^{3}}$ means the parallel curve to $\gamma_{k}$, in $M(\mathcal{J})$, such that the distance between them is $\left.j / N^{3}\right)$.

- $\mathcal{A}_{k}=\bigcup_{j=0}^{N^{2}-1} \overline{\operatorname{Int} \mathcal{G}_{2 j+1, k} \backslash \operatorname{Int} \mathcal{G}_{2 j, k}}$ and $\widetilde{\mathcal{A}}_{k}=\bigcup_{j=1}^{N^{2}} \overline{\operatorname{Int} \mathcal{G}_{2 j, k} \backslash \operatorname{Int} \mathcal{G}_{2 j-1, k}}$.

- $\mathcal{R}_{k}=\bigcup_{j=0}^{2 N^{2}} \mathcal{G}_{j, k}, \mathcal{B}_{k}=\bigcup_{j=1}^{N} L_{2 j, k}$, and $\widetilde{\mathcal{B}}_{k}=\bigcup_{j=0}^{N-1} L_{2 j+1, k}$.

- $\mathcal{L}_{k}=\mathcal{B}_{k} \cap \mathcal{A}_{k}, \widetilde{\mathcal{L}}_{k}=\widetilde{\mathcal{B}}_{k} \cap \widetilde{\mathcal{A}}_{k}$, and $H_{k}=\mathcal{R}_{k} \cup \mathcal{L}_{k} \cup \widetilde{\mathcal{L}}_{k}$.

- $\Omega_{N, k}=\left\{p \in \operatorname{Int}\left(\mathcal{G}_{2 N^{2}, k}\right) \backslash \operatorname{Int}\left(\mathcal{G}_{0, k}\right) \mid \operatorname{dist}_{(M, d s)}\left(p, H_{k}\right) \geq 1 / 4 N^{3}\right\}$.

- $\Omega_{N}=\bigcup_{k=1}^{\mathrm{E}} \Omega_{N, k}$.

- $\omega_{i}^{k}$ is the union of the curve $L_{i, k}$ and those connected components of $\Omega_{N, k}$ that have nonempty intersection with $L_{i, k}$ for $(i, k) \in I$.

- $\varpi_{i}^{k}=\left\{p \in M \mid \operatorname{dist}_{(M, d s)}\left(p, \omega_{i}^{k}\right)<\delta(N)\right\}$, where $\delta(N)>0$ is chosen in such a way that the sets $\bar{\varpi}_{i}^{k},(i, k) \in I$, are pairwise disjoint. 
Now, assume that $N$ is large enough so that the following assertions hold:

(A1) $\operatorname{diam}_{(M, d s)}\left(\varpi_{i}^{k}\right)<$ const $/ N$.

(A2) If $\lambda^{2} \cdot d s^{2}$ is a conformal metric on $\overline{M(\mathcal{J})}$ that satisfies

$$
\lambda \geq \begin{cases}c & \text { in } M(\mathcal{J}), \\ c N^{4} & \text { in } \Omega_{N},\end{cases}
$$

for $c>0$, and if $\alpha$ is a curve in $\overline{M(\mathcal{J})}$ connecting $\gamma_{k}^{\zeta}$ and $\gamma_{k}$, for some $k \in\{1, \ldots, \mathrm{E}\}$, then length ${ }_{\lambda \cdot d s}(\alpha) \geq$ const $c N$.

The next step consists of the construction, for $N$ large enough, of a sequence $\left\{F_{i}^{k} \mid(i, k) \in I\right\}$ of conformal minimal immersions (with boundary) defined on $\overline{M(\mathcal{J})}$. We consider $F_{0}^{1}=X$ and denote $F_{0}^{k}=F_{2 N}^{k-1}, \forall k=2, \ldots$, E. These immersions must be constructed to satisfy the following list of properties:

$\left(1_{i}^{k}\right) F_{i}^{k}(p)=\operatorname{Re}\left(\int_{p_{0}}^{p} \Phi^{i, k}\right)$, where $\Phi^{i, k}=\phi^{i, k} \omega$.

$\left(2_{i}^{k}\right)\left\|\phi^{i, k}(p)-\phi^{i-1, k}(p)\right\| \leq 1 / N^{2}$, for all $p \in \overline{M(\mathcal{J})} \backslash \varpi_{i}^{k}$.

$\left(3_{i}^{k}\right)\left\|\phi^{i, k}(p)\right\| \geq N^{7 / 2}$, for all $p \in \omega_{i}^{k}$.

$\left(4_{i}^{k}\right)\left\|\phi^{i, k}(p)\right\| \geq \mathrm{const} / \sqrt{N}$, for all $p \in \varpi_{i}^{k}$.

$\left(5_{i}^{k}\right) \operatorname{dist}_{\mathbb{S}^{2}}\left(G_{i}^{k}(p), G_{i-1}^{k}(p)\right)<1 / N^{2}$, for all $p \in \overline{M(\mathcal{J})} \backslash \varpi_{i}^{k}$, where $\operatorname{dist}_{\mathbb{S}^{2}}$ is the intrinsic distance in $\mathbb{S}^{2}$ and $G_{i}^{k}$ represents the Gauss map of $F_{i}^{k}$.

$\left(6_{i}^{k}\right)$ There exists an orthonormal basis of $\mathbb{R}^{3}, S_{i}^{k}=\left\{e_{1}, e_{2}, e_{3}\right\}$ such that 1

$\left(6.1_{i}^{k}\right)$ For any $p \in \overline{\varpi_{i}^{k}}$ with $\left\|X(p)-\beta_{k}\left(\mathrm{P}_{k}(p)\right)\right\| \geq 1 / \sqrt{N}$, we have $\|(X(p)-$ $\left.\beta_{k}\left(\mathrm{P}_{k}(p)\right)\right)_{\left(*, S_{i}^{k}\right)} \|<\mathrm{const} / \sqrt{N}$.

$\left(6.2_{i}^{k}\right)\left(F_{i}^{k}(p)\right)_{\left(3, S_{i}^{k}\right)}=\left(F_{i-1}^{k}(p)\right)_{\left(3, S_{i}^{k}\right)}$, for all $p \in \overline{M(\mathcal{J})}$.

$\left(7_{i}^{k}\right)\left\|F_{i}^{k}(p)-F_{i-1}^{k}(p)\right\| \leq \mathrm{const} / N^{2}, \forall p \in \overline{M(\mathcal{J})} \backslash \varpi_{i}^{k}$.

The sequence $\left\{F_{i}^{k} \mid(i, k) \in I\right\}$ is constructed in a recursive way. We construct $F_{i}^{k}$ starting from $F_{i-1}^{k}$ by using a López-Ros transformation. When $i-1=0$ we adopt the convention that $F_{0}^{k}:=F_{2 N}^{k-1}$, if $k>1$, and $F_{0}^{1}:=X$. The same holds for the Weierstrass representations.

Suppose that we have $\left\{F_{j}^{l} \mid(j, l)<(i, k)\right\}$. Assume the existence of the frame $S_{i}^{k}$ satisfying $\left(6.1_{i}^{k}\right)$. Its existence is implied by Properties (A1) and $\left(5_{j}^{l}\right),(j, l)<(i, k)$ (we omit the details on how to obtain it). Let $\left(g^{i-1, k}, \Phi_{3}^{i-1, k}\right)$ be the Weierstrass data of $F_{i-1}^{k}$ in $S_{i}^{k}$. To construct the immersion $F_{i}^{k}$, we consider a positive $\alpha$, and a holomorphic function $h_{\alpha}: \overline{M(\mathcal{J})} \rightarrow \mathbb{C}^{*}$ satisfying:

- $\left|h_{\alpha}-\alpha\right|<1 / \alpha$, in $\omega_{i}^{k}$.

- $\left|h_{\alpha}-1\right|<1 / \alpha$, in $\overline{M(\mathcal{J})} \backslash \varpi_{i}^{k}$.

- The Weierstrass data $\left(g^{i, k}:=g^{i-1, k} / h_{\alpha}, \Phi_{3}^{i, k}:=\Phi_{3}^{i-1, k}\right)$ give a well-defined minimal immersion in $\overline{M(\mathcal{J})}$.

The existence of such a function is known 2, Lemma 1]. Then, for a large enough value of the parameter $\alpha$, the immersion $F_{i}^{k}(p):=\operatorname{Re} \int_{p_{0}}^{p} \Phi^{i, k}$ whose Weierstrass data in $S_{i}^{k}$ are $\left(g^{i, k}, \Phi_{3}^{i, k}\right)$ satisfies the above list of properties. In this way, we have constructed the sequence $\left\{F_{i}^{k} \mid(i, k) \in I\right\}$ satisfying Properties $\left(1_{i}^{k}\right), \ldots,\left(7_{i}^{k}\right)$.

\footnotetext{
${ }^{1}$ Given $v \in \mathbb{R}^{3}$, and $S$ a coordinate system, we let $v_{(k, S)}$ denote the $k$-th coordinate of $v$ in $S$. The first two coordinates of $v$ in this basis are represented by $v_{(*, S)}=\left(v_{(1, S)}, v_{(2, S)}\right)$.
} 
If $N$ is large enough, then the set

$$
\mathcal{D}=\left\{p \in \overline{M(\mathcal{J})} \backslash M\left(\mathcal{J}^{\zeta}\right) \mid s<\operatorname{dist}_{\left(\overline{M(\mathcal{J})}, F_{2 N}^{\mathrm{E}}\right)}\left(p, M\left(\mathcal{J}^{\zeta}\right)\right)<2 s\right\}
$$

is nonempty, and $\mathcal{J}$ and $\mathcal{J}^{\epsilon}$ are contained in different connected components of $\overline{M(\mathcal{J})} \backslash \mathcal{D}$. To check this claim, use Properties (A2), $\left(2_{i}^{k}\right),\left(3_{i}^{k}\right),\left(4_{i}^{k}\right),(i, k) \in I$, and take (2.2) into account. Then, for any multicycle $\widetilde{\mathcal{J}}$ on $\mathcal{D}$, the immersion $\widetilde{X}:=$ $F_{2 N}^{\mathrm{E}}: \overline{M(\widetilde{\mathcal{J}})} \rightarrow \mathbb{R}^{3}$ satisfies the conclusion of Lemma 1 except for (L1,2). Indeed, (L1,1) is implied by $\left(1_{2 N}^{\mathrm{E}}\right)$. Statement (L1 6) trivially follows from $\left(7_{i}^{k}\right),(i, k) \in I$. From $\left(6_{i}^{k}\right),\left(7_{i}^{k}\right),(i, k) \in I$, and the choice of $\widetilde{\mathcal{J}}$, we obtain, in a more elaborate way (we omit the details), (प1 7). The remaining properties follow from the choice of $\widetilde{\mathcal{J}}$ and the positive $\zeta$. Furthermore, it is clear that, up to infinitesimal variations of the cycle $\widetilde{\gamma}_{i} \subset \mathcal{D}$, it can be guaranteed that $\widetilde{X}\left(\widetilde{\gamma}_{i}\right)$ has no self-intersections, $\forall i=1, \ldots, \mathrm{E}$, which proves (L1,2).

\section{Main Lemma}

The next result is the kernel of this paper. It shows that it is possible to perturb a given minimal surface with boundary and finite topology outside a compact set in such a way that the intrinsic diameter of the surface can be increased, while the extrinsic one is preserved. At the same time, one can obtain the embeddedness of the resulting immersion along its boundary curves.

Lemma 2. Let $\mathcal{J}=\left\{\gamma_{1}, \ldots, \gamma_{\mathrm{E}}\right\}$ be a multicycle on $M^{\prime}, X: \overline{M(\mathcal{J})} \rightarrow \mathbb{R}^{3}$ a conformal minimal immersion, and $p_{0} \in M(\mathcal{J})$ with $X\left(p_{0}\right)=0$.

Then, for any $\lambda>0$, and any $\mu>0$ such that $p_{0} \in M\left(\mathcal{J}^{\mu}\right)$, there exist a multicycle $\widehat{\mathcal{J}}=\left\{\widehat{\gamma}_{1}, \ldots, \widehat{\gamma}_{\mathrm{E}}\right\}$ and a conformal minimal immersion $\widehat{X}: \overline{M(\widehat{\mathcal{J}})} \rightarrow \mathbb{R}^{3}$ with the following properties:

(L2,1) $\widehat{X}\left(p_{0}\right)=0$.

(L2,2) $\widehat{X}\left(\widehat{\gamma}_{i}\right)$ is a Jordan curve, for any $i=1, \ldots, \mathrm{E}$.

(L2]3) $\widehat{\gamma}_{i} \subset \mathcal{T}\left(\gamma_{i}\right)$, where $\mathcal{T}\left(\gamma_{i}\right)$ is a tubular neighborhood of $\gamma_{i}$, for $i=1, \ldots$, E.

(L2,4) $\mathcal{J}^{\mu}<\widehat{\mathcal{J}}<\mathcal{J}$.

(L2,5) $\lambda<\operatorname{dist} \frac{}{(M(\widehat{\mathcal{J}}), \widehat{X})}(p, \widehat{\mathcal{J}}), \forall p \in \mathcal{J}^{\mu}$.

(L2,6) $\|\widehat{X}-X\|<\mu$ in $\overline{M(\widehat{\mathcal{J}})}$.

(L2,7) $\delta^{H}(X(\overline{M(\mathcal{J})}), \widehat{X}(\overline{M(\widehat{\mathcal{J}})}))<\mu$.

Proof. Consider $c_{0}<\mu, r_{1}$ and $\rho_{1}$ three positive constants which will be specified later. Define

$$
r_{n}=\sqrt{r_{n-1}^{2}+\left(\frac{2 c_{0}}{n}\right)^{2}}+\frac{c_{0}}{n^{2}}, \quad \rho_{n}=\rho_{1}+\sum_{i=2}^{n} \frac{c_{0}}{i} .
$$

Assume the constants $c_{0}$ and $r_{1}$ are small enough so that

$$
\lim _{n \rightarrow \infty} r_{n}<\frac{\mu}{2}
$$

The next step consists of applying Lemma 1 In order to do it, we need a family $\Sigma_{1}, \ldots, \Sigma_{\mathrm{E}}$, of analytic cycles on $M^{\prime}$. We consider $\Sigma_{i}:=\gamma_{i}, \forall i=1, \ldots$, E. Hence it is clear that $\mathcal{I}=\left\{\Sigma_{1}, \ldots, \Sigma_{\mathrm{E}}\right\}$ is a multicycle on $M^{\prime}$. We also need a family of analytic curves $\beta_{i}: \Sigma_{i} \rightarrow \mathbb{R}^{3}$. Take $\beta_{i}:=X_{\mid \gamma_{i}}, \forall i=1, \ldots$, E. 
For any natural $n$ let us construct a family $\chi_{n}=\left\{\mathcal{J}_{n}, X_{n}, \epsilon_{n}\right\}$ consisting of

$\left(1_{n}\right) \mathcal{J}_{n}=\left\{\gamma_{n, 1}, \ldots, \gamma_{n, \mathrm{E}}\right\}$ is a multicycle with $\gamma_{n, i} \subset \mathcal{T}\left(\Sigma_{i}\right)$ for $i=1, \ldots, \mathrm{E}$.

$\left(2_{n}\right) X_{n}: \overline{M\left(\mathcal{J}_{n}\right)} \rightarrow \mathbb{R}^{3}$ is a conformal minimal immersion with $X_{n}\left(p_{0}\right)=0$, and such that $X_{n}\left(\gamma_{n, i}\right)$ is a Jordan curve, for any $i=1, \ldots, \mathrm{E}$ (only holds for $n \geq 2$ ).

$\left(3_{n}\right)\left\{\epsilon_{n}\right\}_{n \in \mathbb{N}}$ is a decreasing sequence of positive numbers such that $\epsilon_{n}<c_{0} / n^{2}$. Moreover, the sequence $\left\{\chi_{n}\right\}_{n \in \mathbb{N}}$ will satisfy the following properties:

$\left(4_{n}\right) \mathcal{J}^{\mu}<\mathcal{J}_{n-1}^{\epsilon_{n}}<\mathcal{J}_{n}<\mathcal{J}_{n-1}$ (only holds for $n \geq 2$ ).

$\left(5_{n}\right) \rho_{n}<\operatorname{dist}_{\left(\overline{M\left(\mathcal{J}_{n}\right)}, X_{n}\right)}\left(p, \mathcal{J}_{n}\right)$, for all $p \in \mathcal{J}^{\mu}$.

$\left(6_{n}\right)\left\|X_{n}(p)-X\left(\mathrm{P}_{i}(p)\right)\right\|<r_{n}$, for any $p \in \gamma_{n, i}$, for any $i=1, \ldots$, E (recall that $\mathrm{P}_{i}: \mathcal{T}\left(\Sigma_{i}\right) \rightarrow \Sigma_{i}$ is the natural projection).

The sequence will be obtained following an inductive method. For the first family, $\chi_{1}$, we choose $X_{1}=X$ and $\mathcal{J}_{1}=\mathcal{J}$. This choice and the hypotheses of the lemma guarantee that Properties $\left(1_{1}\right)$ and $\left(6_{1}\right)$ are satisfied. Notice that Property $\left(2_{1}\right)$ might not hold. This is not a problem; it will not be used in the construction of the remaining elements of the sequence $\left\{\chi_{n}\right\}_{n \geq 2}$. Let $\rho_{1}$ be small enough such that

$$
\rho_{1}<\operatorname{dist}_{\left(\overline{M\left(\mathcal{J}_{1}\right)}, X_{1}\right)}\left(p, \mathcal{J}_{1}\right), \quad \forall p \in \mathcal{J}^{\mu} .
$$

This choice implies $\left(5_{1}\right)$. Finally, we take $\epsilon_{1}<\min \left\{c_{0}, r_{1}\right\}$. Hence, $\left(3_{1}\right)$ holds. The family $\chi_{1}$ is well defined. Moreover, we assume that $\epsilon_{1}$ is small enough so that $\gamma_{i}^{\epsilon_{1}} \subset \mathcal{T}\left(\Sigma_{i}\right), \forall i=1, \ldots, \mathrm{E}$, and so that given $p$ in the connected component of $\overline{M(\mathcal{J})} \backslash M\left(\mathcal{J}^{\epsilon_{1}}\right)$ around $\gamma_{i}$, for $i=1, \ldots, \mathrm{E}$, then

$$
\left\|X(p)-X\left(\mathrm{P}_{i}(p)\right)\right\|<r_{1}<\frac{\mu}{2} .
$$

This choice is possible since $\mathcal{J}_{1}=\mathcal{J}$ and $X$ is uniformly continuous.

Assume that we have defined $\chi_{1}, \ldots, \chi_{n-1}$. In order to construct $\chi_{n}$ we take a real number $\xi>0$ such that

$$
\rho_{n-1}<\operatorname{dist}_{\left(\overline{M\left(\mathcal{J}_{n-1}\right)}, X_{n-1}\right)}\left(p, \mathcal{J}_{n-1}^{\xi}\right), \quad \forall p \in \mathcal{J}^{\mu} .
$$

Its existence is implied by $\left(5_{n-1}\right)$. Consider $\epsilon_{n}<\min \left\{\epsilon_{n-1}, c_{0} / n^{2}, \xi\right\}$. Hence $\left(3_{n}\right)$ holds. Now, we consider the multicycle $\mathcal{J}_{n}$ and the conformal minimal immersion $X_{n}: \overline{M\left(\mathcal{J}_{n}\right)} \rightarrow \mathbb{R}^{3}$ given by Lemma 1 for the data

$$
X=X_{n-1}, \quad \mathcal{J}=\mathcal{J}_{n-1}, \quad r=r_{n-1}, \quad s=\frac{c_{0}}{n}, \quad \epsilon=\epsilon_{n} .
$$

Recall that $\Sigma_{i}=\gamma_{i}$ and $\beta_{i}=X_{\mid \gamma_{i}}, i=1, \ldots$, E. Then Properties (L1 3), (L11) and (L1,2) in Lemma 1 imply $\left(1_{n}\right)$ and $\left(2_{n}\right) .\left(4_{n}\right)$ follows from (L1, 4$)$ and the fact that $\sum_{k=2}^{n} \epsilon_{k}<\mu$. In order to check $\left(5_{n}\right)$ we assume that $\epsilon_{n}$ was chosen small enough so that

$$
\rho_{n-1}<\operatorname{dist}_{\left(\overline{M\left(\mathcal{J}_{n}\right)}, X_{n}\right)}\left(p, \mathcal{J}_{n-1}^{\xi}\right), \quad \forall p \in \mathcal{J}^{\mu} .
$$

This assumption is possible thanks to equation (3.3), and since (L1 6) guarantees that $X_{n}$ converges to $X_{n-1}$ uniformly on $\overline{M\left(\mathcal{J}_{n-1}^{\xi}\right)}$, as $\epsilon_{n}$ goes to zero. From this inequality, the definition of $\rho_{n}$ and (L1,5) we conclude $\left(5_{n}\right)$. Finally, (L1,7) trivially implies $\left(6_{n}\right)$. In this way, we have constructed the sequence $\left\{\chi_{n}\right\}_{n \in \mathbb{N}}$ satisfying Properties $\left(1_{n}\right), \ldots,\left(6_{n}\right)$.

Let us check that, for $n_{0}$ large enough, the multicycle $\mathcal{J}_{n_{0}}$ and the immersion $X_{n_{0}}$ satisfy the conclusion of Lemma 2. Indeed, Properties $\left(5_{n}\right), n \in \mathbb{N}$, and the 
fact that the sequence $\left\{\rho_{n}\right\}_{n \in \mathbb{N}}$ diverges guarantee the existence of a natural $n_{0}$ such that

$$
\lambda<\operatorname{dist}_{\left(\overline{M\left(\mathcal{J}_{n}\right)}, X_{n}\right)}\left(p, \mathcal{J}_{n}\right), \quad \forall p \in \mathcal{J}^{\mu}, \quad \forall n \geq n_{0} .
$$

Define the multicycle $\widehat{\mathcal{J}}:=\mathcal{J}_{n_{0}}$ and the conformal minimal immersion $\widehat{X}:=X_{n_{0}}$ :

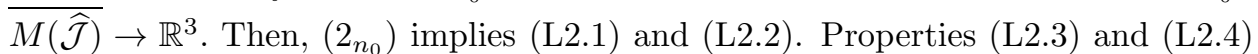
trivially hold from $\left(1_{n_{0}}\right)$ and $\left(4_{n_{0}}\right)$, respectively. Inequality (3.4) implies (L2,5). Finally, taking into account (3.2), $\left(6_{n_{0}}\right)$ and (3.1), we obtain

$$
\begin{gathered}
\|X(p)-\widehat{X}(p)\| \leq\left\|X(p)-X\left(\mathrm{P}_{i}(p)\right)\right\|+\left\|X\left(\mathrm{P}_{i}(p)\right)-\widehat{X}(p)\right\| \\
<\frac{\mu}{2}+r_{n_{0}}<\mu, \quad \forall p \in \widehat{\gamma}_{i}, \quad \forall i=1, \ldots, \mathrm{E} .
\end{gathered}
$$

Hence $\|X(p)-\widehat{X}(p)\|<\mu$ for any $p \in \widehat{\mathcal{J}}$. Therefore the Maximum Principle for harmonic maps guarantees that this inequality holds for any $p \in \overline{M(\widehat{\mathcal{J}})}$. This proves Property (L2,6). Finally, (L2,7) follows from (L26), (3.2) and $\left(6 n_{n_{0}}\right)$.

\section{Compact COmplete minimal immersions in $\mathbb{R}^{3}$}

Now, we are able to prove the theorems stated in the introduction. They follow from the following one.

Theorem 1. Let $\mathcal{J}$ be a multicycle on the Riemann surface $M^{\prime}$, and $\phi: \overline{M(\mathcal{J})} \rightarrow$ $\mathbb{R}^{3}$ a conformal minimal immersion. Then, for any $\epsilon>0$, there exist a domain $M_{\epsilon}$ and a conformal complete minimal immersion $\phi_{\epsilon}: M_{\epsilon} \rightarrow \mathbb{R}^{3}$ satisfying

(I) $\overline{M\left(\mathcal{J}^{\epsilon}\right)} \subset M_{\epsilon} \subset \overline{M_{\epsilon}} \subset M(\mathcal{J})$, and $M_{\epsilon}$ has the same topological type as $M(\mathcal{J})$.

(II) $\phi_{\epsilon}$ admits a continuous extension $\Phi_{\epsilon}: \overline{M_{\epsilon}} \rightarrow \mathbb{R}^{3}$.

(III) $\left\|\phi-\Phi_{\epsilon}\right\|<\epsilon$ in $\overline{M_{\epsilon}}$.

(IV) $\left(\Phi_{\epsilon}\right)_{\mid \Gamma}$ is an embedding, for any $\Gamma$ connected component of $\partial M_{\epsilon}$.

(V) $\delta^{H}\left(\phi(\overline{M(\mathcal{J})}), \Phi_{\epsilon}\left(\overline{M_{\epsilon}}\right)\right)<\epsilon$.

(VI) The Hausdorff dimension of $\Phi_{\epsilon}(\Gamma)$ is 1 , for any $\Gamma$ connected component of $\partial M_{\epsilon}$.

4.1. Proof of Theorem 1. Assume $\mathcal{J}$ consists of e cycles. Label $\varepsilon:=\epsilon / 2$ and consider a multicycle $\mathcal{J}^{\prime}=\left\{\gamma_{1}, \ldots, \gamma_{\mathrm{E}}\right\}$ satisfying

(a) $\mathcal{J}^{\varepsilon}<\mathcal{J}^{\prime}<\mathcal{J}$.

(b) $\phi\left(\gamma_{i}\right)$ is a Jordan curve, for any $i=1, \ldots$, E.

(c) $\delta^{H}\left(\phi(\overline{M(\mathcal{J})}), \phi\left(\overline{M\left(\mathcal{J}^{\prime}\right)}\right)\right)<\varepsilon$.

We use Lemma2 to construct, $\forall n \in \mathbb{N}$, a family $\chi_{n}=\left\{\mathcal{J}_{n}, \mathcal{T}\left(\mathcal{J}_{n}\right), X_{n}, \epsilon_{n}, \xi_{n}, \tau_{n}\right\}$, where

- $\mathcal{J}_{n}=\left\{\gamma_{n, 1}, \ldots, \gamma_{n, \mathrm{E}}\right\}$ is a multicycle on $M^{\prime}$.

- $\mathcal{T}\left(\mathcal{J}_{n}\right)=\left\{\mathcal{T}\left(\gamma_{n, 1}\right), \ldots, \mathcal{T}\left(\gamma_{n, \mathrm{E}}\right)\right\}$, where $\mathcal{T}\left(\gamma_{n, i}\right)$ is a tubular neighborhood of $\gamma_{n, i}$ in $\overline{M\left(\mathcal{J}_{n}\right)}$, for $i=1, \ldots$, E.

- $X_{n}: \overline{M\left(\mathcal{J}_{n}\right)} \rightarrow \mathbb{R}^{3}$ is a conformal minimal immersion.

- $\left\{\epsilon_{n}\right\}_{n \in \mathbb{N}}$ and $\left\{\xi_{n}\right\}_{n \in \mathbb{N}}$ are decreasing sequences of positive real numbers with $\xi_{n}<\epsilon_{n}<6 \varepsilon / \pi^{2} n^{2}$.

- $\left\{\tau_{n}\right\}_{n \in \mathbb{N}}$ is an increasing sequence of positive numbers which diverges to $+\infty$. 
Moreover, we will construct the sequence $\left\{\chi_{n}\right\}_{n \in \mathbb{N}}$ so that the following list of properties holds:

$\left(\mathrm{A}_{n}\right) X_{n}\left(\gamma_{n, i}\right)$ is a Jordan curve, for any $i=1, \ldots, \mathrm{E}$.

$\left(\mathrm{B}_{n}\right) \mathcal{T}\left(\gamma_{n, i}\right) \subset \mathcal{T}\left(\gamma_{n-1, i}\right), \forall i=1, \ldots, \mathrm{E}$.

$\left(\mathrm{C}_{n}\right) \mathcal{J}_{n}^{\xi_{n}} \subset \mathcal{T}\left(\mathcal{J}_{n}\right)$, i.e., $\gamma_{n, i}^{\xi_{n}} \subset \mathcal{T}\left(\gamma_{n, i}\right)$, for any $i=1, \ldots$, E.

$\left(\mathrm{D}_{n}\right) \mathcal{J}_{n-1}^{\xi_{n-1}}<\mathcal{J}_{n-1}^{\epsilon_{n}}<\mathcal{J}_{n}^{\xi_{n}}<\mathcal{J}_{n}<\mathcal{J}_{n-1}$.

$\left(\mathrm{E}_{n}\right)\left\|X_{n}-X_{n-1}\right\|<\epsilon_{n}$ in $\overline{M\left(\mathcal{J}_{n}\right)}$.

$\left(\mathrm{F}_{n}\right)\left(X_{n}\right)_{\mid \overline{\mathcal{T}\left(\gamma_{n, i}\right)}}: \overline{\mathcal{T}\left(\gamma_{n, i}\right)} \rightarrow \mathbb{R}^{3}$ is an embedding, for any $i=1, \ldots, \mathrm{E}$.

$\left(\mathrm{G}_{n}\right)\left\|X_{n}-X_{n-1}\right\|<\min \left\{\eta_{n-1}, 1 /\left(n^{2} \tau_{n-1}^{n}\right)\right\}$, in $\overline{\mathcal{T}\left(\mathcal{J}_{n}\right)} \subset \overline{M\left(\mathcal{J}_{n}\right)}$, where

$$
\eta_{n-1}:=\min \left\{\Psi\left(\overline{\mathcal{T}\left(\gamma_{n-1, i}\right)},\left(X_{n-1}\right)_{\mid \overline{\mathcal{T}\left(\gamma_{n-1, i}\right)}}, n\right) \mid i=1, \ldots, \mathrm{E}\right\} .
$$

Recall the definition of $\Psi$ in Definition 4 above.

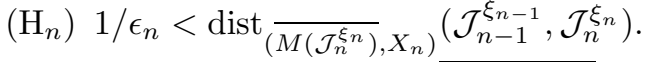

$\left(\mathrm{I}_{n}\right) d s_{X_{n}} \geq \alpha_{n} \cdot d s_{X_{n-1}}$ in $M\left(\mathcal{J}_{n-1}^{\xi_{n-1}}\right)$, where the sequence $\left\{\alpha_{k}\right\}_{k \in \mathbb{N}}$ is given by

$$
\alpha_{1}:=\frac{1}{2} e^{1 / 2}, \quad \alpha_{k}:=e^{-1 / 2^{k}} \text { for } k>1 .
$$

Notice that $0<\alpha_{k}<1$ and $\left\{\prod_{m=1}^{k} \alpha_{m}\right\}_{k \in \mathbb{N}}$ converges to $1 / 2$.

$\left(\mathrm{J}_{n}\right) \delta^{H}\left(X_{n}\left(\overline{M\left(\mathcal{J}_{n}\right)}\right), X_{n-1}\left(\overline{M\left(\mathcal{J}_{n-1}\right)}\right)\right)<\epsilon_{n}$.

$\left(\mathrm{K}_{n}\right) \forall i=1, \ldots, \mathrm{E}$, there exist points $\alpha_{1, i}^{n}, \ldots, \alpha_{N_{n}, i}^{n} \in X_{n}\left(\gamma_{n, i}\right)$ such that

$$
X_{n}\left(\overline{\mathcal{T}\left(\gamma_{n, i}\right)}\right) \subset \bigcup_{j=1}^{N_{n}} \mathbb{B}\left(\alpha_{j, i}^{n}, s_{n}\right),
$$

where $s_{n}=1 / \tau_{n}^{n}, N_{n}$ is the integer part of $\tau_{n} / s_{n}$, and $\mathbb{B}\left(\alpha_{j, i}^{n}, s_{n}\right)=\{y \in$ $\left.\mathbb{R}^{3} \mid\left\|\alpha_{j, i}^{n}-y\right\|<s_{n}\right\}$.

For the first family we choose $\mathcal{J}_{1}=\mathcal{J}^{\prime}$ and $X_{1}=\phi$. Hence Property (b) of $\mathcal{J}^{\prime}$ guarantees $\left(\mathrm{A}_{1}\right)$. Notice that we can choose $\mathcal{T}\left(\mathcal{J}_{1}\right)$ close enough to $\mathcal{J}_{1}$ so that $\left(\mathrm{F}_{1}\right)$ holds. This is possible because of $\left(\mathrm{A}_{1}\right)$ and the local injectivity of $X_{1}$. Define

$$
\tau_{1}:=\max \left\{1, \max \left\{\operatorname{length}\left(X_{1}\left(\gamma_{1, i}\right)\right) \mid i=1, \ldots, \mathrm{E}\right\}\right\} .
$$

For $i=1, \ldots, \mathrm{E}$, let $\alpha_{1, i}^{1}, \ldots, \alpha_{N_{1}, i}^{1}$ be points on $X_{1}\left(\gamma_{1, i}\right)$ with mutual distance along $X_{1}\left(\gamma_{1, i}\right)$. The choice of $\tau_{1}$ implies that this distance is equal to or smaller than $s_{1}$. Hence, the union of $\mathbb{B}\left(\alpha_{j, i}^{1}, s_{1}\right)$ is an open set in $\mathbb{R}^{3}$ which covers the curve $X_{1}\left(\gamma_{1, i}\right)$. Therefore we can choose $\mathcal{T}\left(\mathcal{J}_{1}\right)$ close enough to $\mathcal{J}_{1}$ so that $\left(\mathrm{K}_{1}\right)$ holds. Finally, consider positive numbers $\xi_{1}<\epsilon_{1}<6 \varepsilon / \pi^{2}$, where $\xi_{1}$ is small enough so that $\left(\mathrm{C}_{1}\right)$ holds. Properties $\left(\mathrm{B}_{1}\right),\left(\mathrm{D}_{1}\right),\left(\mathrm{E}_{1}\right),\left(\mathrm{G}_{1}\right),\left(\mathrm{H}_{1}\right),\left(\mathrm{I}_{1}\right)$ and $\left(\mathrm{J}_{1}\right)$ do not make sense.

Assume that we have constructed $\chi_{1}, \ldots, \chi_{n}$. In order to define $\chi_{n+1}$ we consider a sequence $\left\{\widetilde{\epsilon}_{m}\right\}_{m \in \mathbb{N}}$ decreasing to zero with

$$
\widetilde{\epsilon}_{m}<\min \left\{\frac{6 \varepsilon}{\pi^{2}(n+1)^{2}}, \eta_{n}, \frac{1}{\tau_{n}^{n+1}(n+1)^{2}}\right\}, \quad \forall m \in \mathbb{N} .
$$

Notice that $\left(\mathrm{F}_{n}\right)$ guarantees that $\eta_{n}>0$, so the choice of $\widetilde{\epsilon}_{m}$ is possible. Now, apply Lemma 2 to the data

$$
\mathcal{J}=\mathcal{J}_{n}, \quad X=X_{n}, \quad \lambda=\frac{1}{\widetilde{\epsilon}_{m}}, \quad \mu=\widetilde{\epsilon}_{m}
$$


Hence, for any $m \in \mathbb{N}$, we obtain a multicycle $\Gamma_{m}$, and a conformal minimal immersion $Y_{m}: \overline{M\left(\Gamma_{m}\right)} \rightarrow \mathbb{R}^{3}$. Statements (L2 4) and (L2 6) in Lemma 2 imply that, if $m$ is large enough, $\mathcal{J}_{n}^{\xi_{n}}<\Gamma_{m}$ and the sequence $\left\{Y_{m}\right\}_{m \in \mathbb{N}}$ uniformly converges in $\overline{M\left(\mathcal{J}_{n}^{\xi_{n}}\right)}$ to $X_{n}$. In particular, the sequence $\left\{d s_{Y_{m}}\right\}_{m \in \mathbb{N}}$ uniformly converges in $\overline{M\left(\mathcal{J}_{n}^{\xi_{n}}\right)}$ to $d s_{X_{n}}$. Therefore there exists $m_{0} \in \mathbb{N}$ large enough so that

$$
\begin{gathered}
\mathcal{J}_{n}^{\xi_{n}}<\mathcal{J}_{n}^{\widetilde{\epsilon}_{m_{0}}}<\Gamma_{m_{0}}, \\
d s_{Y_{m_{0}}} \geq \alpha_{n+1} \cdot d s_{X_{n}} \quad \text { in } \overline{M\left(\mathcal{J}_{n}^{\xi_{n}}\right)} .
\end{gathered}
$$

Define $\mathcal{J}_{n+1}:=\Gamma_{m_{0}}, X_{n+1}:=Y_{m_{0}}$, and $\epsilon_{n+1}:=\widetilde{\epsilon}_{m_{0}}$. Hence Statement (L2) 2) guarantees $\left(\mathrm{A}_{n+1}\right)$. Then we choose $\mathcal{T}\left(\mathcal{J}_{n+1}\right)$, a tubular neighborhood of $\mathcal{J}_{n+1}$ in $\overline{M\left(\mathcal{J}_{n+1}\right)}$, close enough to $\mathcal{J}_{n+1}$ so that $\left(\mathrm{B}_{n+1}\right)$ and $\left(\mathrm{F}_{n+1}\right)$ hold. This choice is possible because of (L2]3), $\left(\mathrm{A}_{n+1}\right)$ and the local injectivity of $X_{n+1}$. Define

$$
\tau_{n+1}:=\max \left\{\tau_{n}+1, \max \left\{\operatorname{length}\left(X_{n+1}\left(\gamma_{n+1, i}\right)\right) \mid i=1, \ldots, \mathrm{E}\right\}\right\} .
$$

For $i=1, \ldots, \mathrm{E}$, let $\alpha_{1, i}^{n+1}, \ldots, \alpha_{N_{n+1}, i}^{n+1}$ be points on $X_{n+1}\left(\gamma_{n+1, i}\right)$ with mutual distance along $X_{n+1}\left(\gamma_{n+1, i}\right)$. The choice of $\tau_{n+1}$ implies that this distance is not larger than $s_{n+1}$. Therefore the union of $\mathbb{B}\left(\alpha_{j, i}^{n+1}, s_{n+1}\right)$ covers the curve $X_{n+1}\left(\gamma_{n+1, i}\right)$. Hence we can choose $\mathcal{T}\left(\mathcal{J}_{n+1}\right)$ close enough to $\mathcal{J}_{n+1}$ so that $\left(\mathrm{K}_{n+1}\right)$ holds.

Now, taking into account (4.2) and (L2.5), we conclude that

$$
\frac{1}{\epsilon_{n+1}}<\operatorname{dist}_{\left(\overline{M\left(\mathcal{J}_{n+1}\right)}, X_{n+1}\right)}\left(\mathcal{J}_{n}^{\xi_{n}}, \mathcal{J}_{n+1}\right) \text {. }
$$

Then we can choose $\left.\xi_{n+1} \in\right] 0, \epsilon_{n+1}$ [ small enough so that $\left(\mathrm{C}_{n+1}\right),\left(\mathrm{D}_{n+1}\right)$ and $\left(\mathrm{H}_{n+1}\right)$ hold. Moreover, (4.3) implies $\left(\mathrm{I}_{n+1}\right)$. (L26) and (4.1) imply $\left(\mathrm{E}_{n+1}\right)$ and $\left(\mathrm{G}_{n+1}\right)$. Finally (L2,7) guarantees $\left(\mathrm{J}_{n+1}\right)$. In this way we have finished the construction of the sequence $\left\{\chi_{n}\right\}_{n \in \mathbb{N}}$.

Now, define the set

$$
M_{\epsilon}:=\bigcup_{n \in \mathbb{N}} M\left(\mathcal{J}_{n}^{\epsilon_{n+1}}\right)=\bigcup_{n \in \mathbb{N}} M\left(\mathcal{J}_{n}^{\xi_{n}}\right) .
$$

From $\left(\mathrm{D}_{n}\right), n \in \mathbb{N}$, we conclude that the set $M_{\epsilon}$ is an expansive union of domains with the same topological type as $M(\mathcal{J})$. Therefore elementary topological arguments give us that $M_{\epsilon}$ is a domain with the same topological type as $M(\mathcal{J})$. On the other hand, $\left(\mathrm{D}_{n}\right), n \in \mathbb{N}$, also imply that

$$
\overline{M_{\epsilon}}=\bigcap_{n \in \mathbb{N}} \overline{M\left(\mathcal{J}_{n}\right)} .
$$

Then Properties $\left(\mathrm{E}_{n}\right), n \in \mathbb{N}$, guarantee that $\left\{X_{n}\right\}_{n \in \mathbb{N}}$ is a Cauchy sequence uniformly in $\overline{M_{\epsilon}}$ of continuous maps. Therefore it converges to a continuous map $\Phi_{\epsilon}: \overline{M_{\epsilon}} \rightarrow \mathbb{R}^{3}$. Define $\phi_{\epsilon}:=\left(\Phi_{\epsilon}\right)_{\mid M_{\epsilon}}: M_{\epsilon} \rightarrow \mathbb{R}^{3}$.

Let us check that $\phi_{\epsilon}$ satisfies the conclusion of the theorem.

- From Properties $\left(\mathrm{I}_{n}\right), n \in \mathbb{N}, \phi_{\epsilon}$ is a conformal minimal immersion.

- Completeness of $\phi_{\epsilon}$ follows from Properties $\left(\mathrm{H}_{n}\right),\left(\mathrm{I}_{n}\right)$ and the fact that the sequence $\left\{1 / \epsilon_{n}\right\}_{n \in \mathbb{N}}$ diverges.

- Statements (I) and (II) trivially hold (in order to check (I), take into account Property (a) of $\mathcal{J}^{\prime}$ ). 
- Notice that $\sum_{n=1}^{\infty} \epsilon_{n}<\varepsilon=\epsilon / 2$. Taking this fact into account, Statement (III) follows from $\left(\mathrm{E}_{n}\right), n \in \mathbb{N}$, and Statement $(\mathrm{V})$ is implied by $\left(\mathrm{J}_{n}\right), n \in \mathbb{N}$, and Property (c) of the multicycle $\mathcal{J}^{\prime}$.

- In order to prove (IV) we have to work a little further. From Statement (I) we obtain that $\partial M_{\epsilon}$ has exactly E connected components, one of them in each connected component of $M(\mathcal{J}) \backslash M\left(\mathcal{J}^{\epsilon}\right)$. Consider two different points, $p$ and $q$, in the same connected component $\Gamma$ of $\partial M_{\epsilon}$. Assume that $\Gamma$ is the connected component of $\partial M_{\epsilon}$ related with the $i$-th cycle of $\mathcal{J}$. Then Properties $\left(\mathrm{B}_{n}\right),\left(\mathrm{D}_{n}\right), n \in \mathbb{N}$, and the definition of $M_{\epsilon}$ imply that

$$
\{p, q\} \subset \Gamma \subset \overline{\mathcal{T}\left(\gamma_{n, i}\right)}, \quad \forall n \in \mathbb{N} .
$$

On the other hand, there exists $n_{0} \in \mathbb{N}$ such that

$$
\operatorname{dist}_{\left(M^{\prime}, d s\right)}(p, q)>1 / n, \quad \forall n \geq n_{0} .
$$

Then, for any $n>n_{0}$, from Property $\left(\mathrm{G}_{n}\right)$ and the definition of the constant $\eta_{n-1}$, we have

$$
\begin{gathered}
\left\|X_{n-1}(p)-X_{n-1}(q)\right\| \\
\leq\left\|X_{n-1}(p)-X_{n}(p)\right\|+\left\|X_{n-1}(q)-X_{n}(q)\right\|+\left\|X_{n}(p)-X_{n}(q)\right\| \\
<2 \eta_{n-1}+\left\|X_{n}(p)-X_{n}(q)\right\| \\
\leq \frac{1}{n^{2}} \cdot\left\|X_{n-1}(p)-X_{n-1}(q)\right\|+\left\|X_{n}(p)-X_{n}(q)\right\|,
\end{gathered}
$$

where we have taken (4.4) and (4.5) into account to bound $\eta_{n-1}$. Hence

$$
\left\|X_{n}(p)-X_{n}(q)\right\|>\left(1-\frac{1}{n^{2}}\right) \cdot\left\|X_{n-1}(p)-X_{n-1}(q)\right\|, \quad \forall n>n_{0} .
$$

Then, for any $k>0$, the above inequalities guarantee that

$$
\left\|X_{n_{0}+k}(p)-X_{n_{0}+k}(q)\right\|>\left\|X_{n_{0}}(p)-X_{n_{0}}(q)\right\| \cdot \prod_{m=n_{0}+1}^{n_{0}+k}\left(1-\frac{1}{m^{2}}\right) .
$$

If we take limits in (4.6) as $k \rightarrow \infty$, then we obtain that

$$
\left\|\Phi_{\epsilon}(p)-\Phi_{\epsilon}(q)\right\| \geq \frac{1}{2}\left\|X_{n_{0}}(p)-X_{n_{0}}(q)\right\|>0
$$

where we have taken into account $\left(\mathrm{F}_{n_{0}}\right)$. This inequality proves Statement (IV).

- Finally, let us check Statement (VI). Fix $n \in \mathbb{N}$ so that $\sum_{k=n}^{\infty} 1 / k^{2}<1$. Let $\Gamma$ be the connected component of $\partial M_{\epsilon}$ related with the $i$-th cycle of $\mathcal{J}$, and consider $p \in \Gamma$. Notice that $p \in \overline{\mathcal{T}\left(\gamma_{m, i}\right)}, \forall m \in \mathbb{N}$. Then, for any $k>n$, from $\left(\mathrm{G}_{m}\right)$, $m=n+1, \ldots, k$, we deduce that

$$
\left\|X_{k}(p)-X_{n}(p)\right\| \leq \frac{1}{k^{2} \tau_{k-1}^{k}}+\frac{1}{(k-1)^{2} \tau_{k-2}^{k-1}}+\cdots+\frac{1}{(n+1)^{2} \tau_{n}^{n+1}} .
$$

Then, using that $\left\{\tau_{m}\right\}_{m \in \mathbb{N}}$ is increasing and $\tau_{n} \geq 1$, we obtain

$$
\left\|X_{k}(p)-X_{n}(p)\right\|<\left(\sum_{m=n+1}^{k} \frac{1}{m^{2}}\right) \frac{1}{\tau_{n}^{n}}<\frac{1}{\tau_{n}^{n}} .
$$

If we take limits in (4.7) as $k$ goes to infinity, then we have

$$
\left\|\Phi_{\epsilon}(p)-X_{n}(p)\right\|<\frac{1}{\tau_{n}^{n}}=s_{n}, \quad \text { for any } n \text { large enough. }
$$


On the other hand, Property $\left(\mathrm{K}_{n}\right)$ guarantees that

$$
X_{n}(p) \in \bigcup_{j=1}^{N_{n}} \mathbb{B}\left(\alpha_{j, i}^{n}, s_{n}\right), \quad \forall p \in \Gamma .
$$

Hence, taking into account (4.8), we have

$$
\Phi_{\epsilon}(\Gamma) \subset \bigcup_{j=1}^{N_{n}} \mathbb{B}\left(\alpha_{j, i}^{n}, 2 s_{n}\right) .
$$

Moreover, it is clear that $N_{n} \cdot\left(2 s_{n}\right)^{1+1 / n}<4$. This inequality particularly means that the Hausdorff measure $\mathcal{H}^{1}\left(\Phi_{\epsilon}(\Gamma)\right)<\infty$ and so the Hausdorff dimension of $\Phi_{\epsilon}(\Gamma)$ is at most 1 (see [13] for details about Hausdorff measure). However, the Hausdorff dimension of $\Phi_{\epsilon}(\Gamma)$ is at least 1 (it is a curve).

This proves Statement (VI) and concludes the proof of the theorem.

4.2. Some consequences of Theorem 1, In this subsection we mention some results that follow straightforwardly from Theorem 1 .

The first one is an improvement of Theorem 1, We can trivially refine the behavior of the limit set to obtain the embeddedness of $\Phi_{\epsilon}$ along $\partial M_{\epsilon}$.

Corollary 2. Let $\mathcal{J}$ be a multicycle on the Riemann surface $M^{\prime}$, and $\phi: \overline{M(\mathcal{J})} \rightarrow$ $\mathbb{R}^{3}$ a conformal minimal immersion. Then, for any $\epsilon>0$, there exist a domain $M_{\epsilon}$ and a conformal complete minimal immersion $\phi_{\epsilon}: M_{\epsilon} \rightarrow \mathbb{R}^{3}$ satisfying:

- $\overline{M\left(\mathcal{J}^{\epsilon}\right)} \subset M_{\epsilon} \subset \overline{M_{\epsilon}} \subset M(\mathcal{J})$, and $M_{\epsilon}$ has the same topological type as $M(\mathcal{J})$.

- $\phi_{\epsilon}$ admits a continuous extension $\Phi_{\epsilon}: \overline{M_{\epsilon}} \rightarrow \mathbb{R}^{3}$.

- $\left\|\phi-\Phi_{\epsilon}\right\|<\epsilon$ in $\overline{M_{\epsilon}}$.

- $\left(\Phi_{\epsilon}\right)_{\mid \partial M_{\epsilon}}$ is an embedding.

- $\delta^{H}\left(\phi(\overline{M(\mathcal{J})}), \Phi_{\epsilon}\left(\overline{M_{\epsilon}}\right)\right)<\epsilon$.

- The Hausdorff dimension of $\Phi_{\epsilon}\left(\partial M_{\epsilon}\right)$ is 1 .

The next result is a density type theorem. We prove that the set of finite families of curves in $\mathbb{R}^{3}$ spanned by complete (connected) minimal surfaces is dense in the set of finite families of curves spanned by (connected) minimal surfaces, with the Hausdorff metric.

Corollary 3. Let $\Sigma$ be a finite family of closed curves in $\mathbb{R}^{3}$ so that the Plateau problem for $\Sigma$ admits a solution. Then, for any $\xi>0$, there exist a compact Riemann surface $\mathcal{M}$, an open domain $M \subset \mathcal{M}$ and a continuous map $\Phi: \bar{M} \rightarrow \mathbb{R}^{3}$ such that:

- $\Phi_{\mid M}$ is a conformal complete minimal immersion.

- $\Phi_{\mid \partial M}$ is an embedding.

- $\delta^{H}(\Sigma, \Phi(\partial M))<\xi$.

- The Hausdorff dimension of $\Phi(\partial M)$ is 1 .

The next result shows that compact complete minimal immersions are not rare. Before stating it, recall that any Riemann surface with finite topology and analytic boundary can be seen as the closure of an open region of a compact Riemann surface 1].

Corollary 4. Complete minimal surfaces are dense in the space of minimal surfaces spanning a finite set of closed curves in $\mathbb{R}^{3}$, endowed with the topology of the Hausdorff distance. 
Theorem 10 can be seen as an improvement of the following result [2, Theorem $2]$.

Corollary 5. Let $\mathcal{J}$ be a multicycle on $M^{\prime}$ and $\phi: \overline{M(\mathcal{J})} \rightarrow \mathbb{R}^{3}$ a conformal minimal immersion. Then, for any $\epsilon>0$, there exists a domain $M_{\epsilon}$ in $M^{\prime}$, with $\overline{M(\mathcal{J})} \subset M_{\epsilon}$, and there exists a conformal complete minimal immersion $\phi_{\epsilon}: M_{\epsilon} \rightarrow$ $\mathbb{R}^{3}$ such that

- $\left\|\phi_{\epsilon}-\phi\right\|<\epsilon$ in $\overline{M(\mathcal{J})}$;

- $\delta^{H}\left(\phi(\overline{M(\mathcal{J})}), \overline{\phi_{\epsilon}\left(M_{\epsilon}\right)}\right)<\epsilon$.

\section{ACKNOWLEDGMENTS}

The author is indebted to Francisco Martín for some informative conversations on the paper 12 .

\section{REFERENCES}

1. L. V. Ahlfors and L. Sario, Riemann Surfaces. Princeton University Press, Princeton, New Jersey (1974). MR0114911 (22:5729)

2. A. Alarcón, L. Ferrer and F. Martín, Density theorems for complete minimal surfaces in $\mathbb{R}^{3}$. Geom. Funct. Anal. 18 (1) (2008), 1-49. MR2399094

3. A. Alarcón and N. Nadirashvili, Limit sets for complete minimal immersions. Math. Z. 258 (1) (2008), 107-113. MR2350037 (2008h:53006)

4. E. Calabi, Problems in differential geometry. Ed. S. Kobayashi and J. Ells, Jr., Proceedings of the United States-Japan Seminar in Differential Geometry, Kyoto, Japan, 1965. Nippon Hyoronsha Co., Ltd., Tokyo 170 (1966). MR0216513 (35:7346)

5. T. H. Colding and W. P. Minicozzi, The Calabi-Yau conjectures for embedded surfaces. Ann. of Math. (2) 167 (1) (2008), 211-243. MR2373154 (2008k:53014)

6. J. Douglas, Solution of the problem of Plateau. Trans. Amer. Math. Soc. 33 (1) (1931), 263321. MR1501590

7. L. Ferrer, F. Martín and W. H. Meeks III, Existence of proper minimal surfaces of arbitrary topological type. Preprint.

8. F. J. López, F. Martín and S. Morales, Adding handles to Nadirashvili's surfaces. J. Diff. Geom. 60 (1) (2002), 155-175. MR.1924594 (2003f:53013)

9. F. Martín, W. H. Meeks III and N. Nadirashvili, Bounded domains which are universal for minimal surfaces. Amer. J. Math. 129 (2) (2007), 455-461. MR2306042 (2008b:53010)

10. F. Martín and S. Morales, On the asymptotic behavior of a complete bounded minimal surface in $\mathbb{R}^{3}$. Trans. Amer. Math. Soc. 356 (10) (2004), 3985-3994. MR.2058515 (2005b:53013)

11. F. Martín and S. Morales, Complete proper minimal surfaces in convex bodies of $\mathbb{R}^{3}$ (II): The behavior of the limit set. Comment. Math. Helv. 81 (3) (2006), 699-725. MR 2250860 (2007e:53005)

12. F. Martín and N. Nadirashvili, A Jordan curve spanned by a complete minimal surface. Arch. Ration. Mech. Anal. 184 (2) (2007), 285-301. MR2299764 (2008d:53008)

13. F. Morgan, Geometric Measure Theory. A Beginner's Guide. Third edition. Academic Press, Inc., San Diego, CA (2000). MR1775760 (2001j:49001)

14. N. Nadirashvili, Hadamard's and Calabi-Yau's conjectures on negatively curved and minimal surfaces. Invent. Math. 126 (3) (1996), 457-465. MR1419004 (98d:53014)

15. N. Nadirashvili, An application of potential analysis to minimal surfaces. Mosc. Math. J. 1 (4) (2001), 601-604. MR1901078 (2003f:53014)

16. T. Radó, On Plateau's problem. Ann. of Math. (2) 31 (3) (1930), 457-469. MR.1502955

Departamento de Geometría y Topología, Universidad de Granada, E-18071 Granada, SPAIN

Current address: Departamento de Matemática Aplicada, Universidad de Murcia, E-30100

Espinardo, Murcia, Spain

E-mail address: ant.alarcon@um.es 